Current Developments in Mathematics, 2000

Pages $233-253$

\title{
Scaling limits of random processes and the outer boundary of planar Brownian motion
}

\author{
Oded Schramm
}

\section{Scaling limits and the Stochastic Löwner Evolution}

1.1. Scaling Limits. Often Scaling limits exhibit more symmetry and are more canonical than the discrete models.

Example. Consider the simple random walk on the grid $\mathbb{Z}^{2}$. If you rescale the grid (and rescale time appropriately), then the simple random walk converges to Brownian motion.

In simple random walk (SRW), at each step you are at some vertex and walk to one of the neighbors at random with equal probability.

The scaling is as follows. Let $X_{s}(t):=X\left(s^{2} t\right) / s$, where $X(t)$ is the position of the SRW at time $t$. Then $X_{s}(t)$ converges to Brownian motion, in the appropriate topology, as $s \rightarrow \infty$.

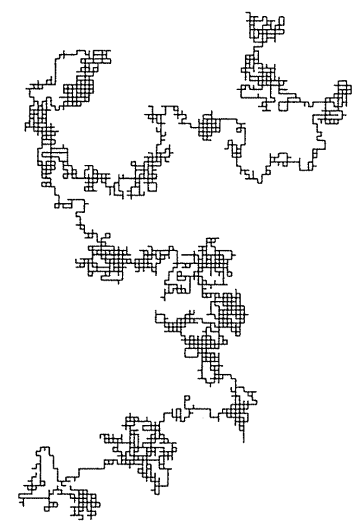

Brownian motion is invariant under rotations, although the simple random walk is not.

Actually, in two dimensions, BM is conformally invariant (up to time-change).

1.2. Conformal invariance. A conformal map $f: D \rightarrow D^{\prime}$ is a homeomorphism from $D$ onto $D^{\prime}$ which is complex-differentiable.

Equivalently, $f$ preserves angles. 


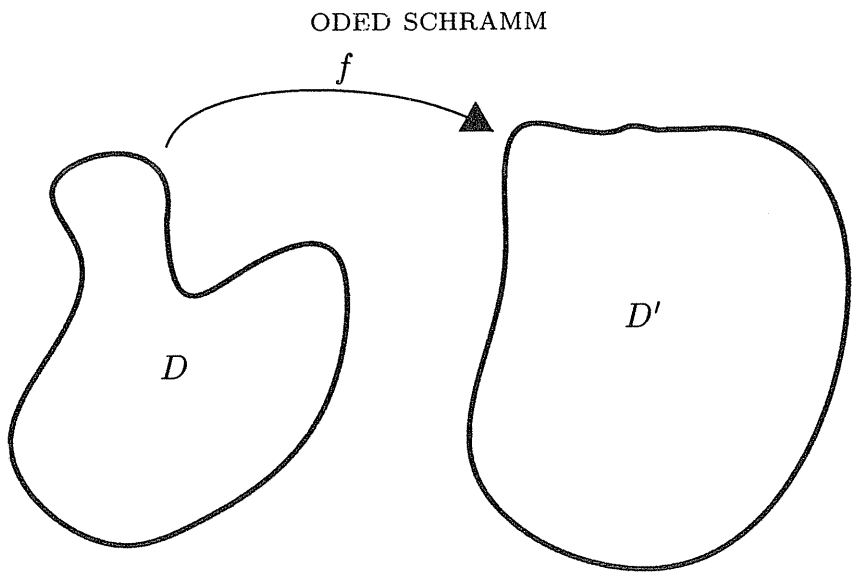

A random process is conformally invariant, if the image of the process under a conformal map $f: D \rightarrow D^{\prime}$ has the same distribution as the process in $D^{\prime}$.

Example. Run Brownian motion $B$ from $p \in D$ until you hit the boundary of $D$. Take the trace (image).

One may consider $f(B)$ as a path from $f(p)$ to $\partial D^{\prime}$. Conformal invariance means that up to reparameterization, $f(B)$ is the same as BM in $D^{\prime}$ from $f(p)$ to $\partial D^{\prime}$.

The above is not a precise definition of what conformal invariance means. Rather, it should serve as a guiding principle. It is perhaps more instructive at this point not to present an exact definition.

1.3. Percolation. Here is one of several models for critical percolation. 


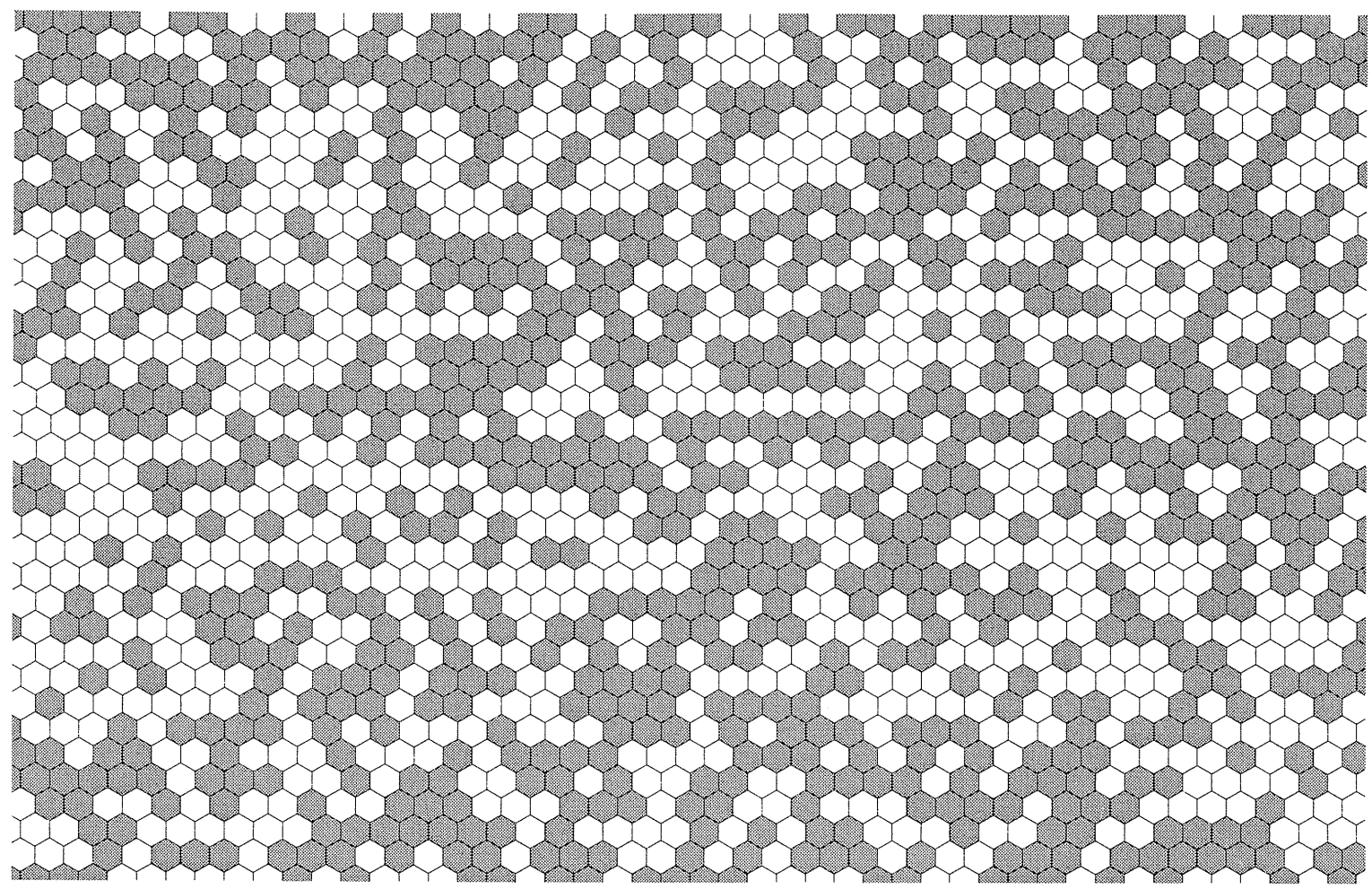

Each hexagon is white (open) with probability $1 / 2$, independently. The connected components of the white regions are studied.

More generally, given $p \in[0,1]$, we may consider $\operatorname{Bernoulli}(p)$ percolation, where each hexagon is white with probability $p$.

There are various other percolation models. A favorite is $\operatorname{Bernoulli}(p)$ bond percolation where each edge of the grid in $\mathbb{Z}^{d}$ is kept with probability $p$ and removed with probability $1-p$.

Harris [Har60] showed that with probability 1 Bernolli(1/2) bond percolation on $\mathbb{Z}^{2}$ has no infinite components, while Kesten [Kes80] showed that when $p>1 / 2$ there are infinite components with probability 1 . The same statements are also known for the percolation model discussed above. The critical parameter $p_{c}=p_{c}(d)$ is defined as the supremum of $p \in[0,1]$ such that with probability 1 there are no infinite components for $\operatorname{Bernoulli}(p)$ bound percolation on $\mathbb{Z}^{d}$. For $d>2$, the value of $p_{c}(d)$ is not known precisely.

There are many interesting works on percolation in 2 and higher dimensions. One problem is to show that there are no infinite components for critical (i.e., $p=$ $p_{c}$ ) Bernoulli bond percolation in $\mathbb{Z}^{d}$. This is known only for $d=2$ (Kesten [ $\left.\operatorname{Kes} 80\right]$ ) and $d \geqslant 19$ (Hara and Slade [HS90]).

1.4. Cardy's formula (Carleson's version). Take an equilateral triangle with unit length edges. Let $x \in[0,1]$. From the upper corner, mark an arc of length $x$ along the right edge. Put a grid of very small hexagons, sample a critical percolation configuration, and consider the event that inside the triangle there is a crossing from the lower edge to the marked arc inside white hexagons. It is a 
conjecture that for every $x \in[0,1]$ the probability of this event tends to $x$ as the mesh of the grid goes to zero.

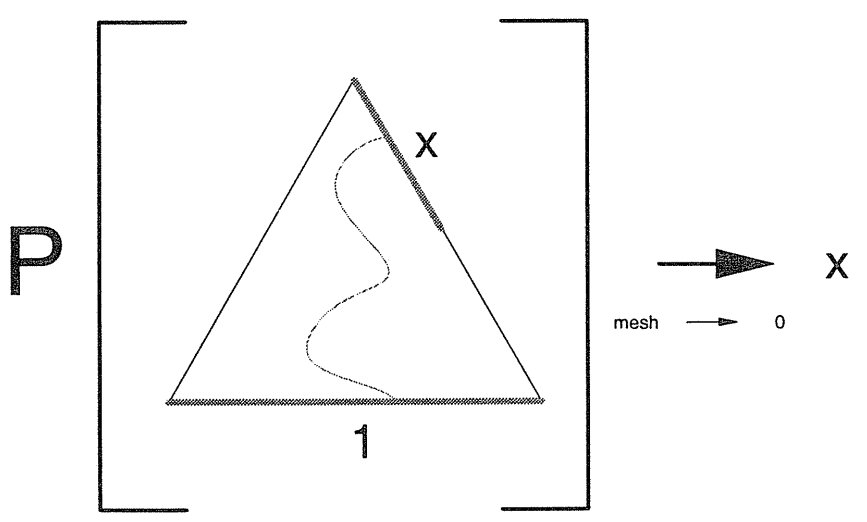

Mathematically speaking, this is a conjecture (Cardy is a physicist). Cardy's reasoning [Car92] uses Conformal field theory and the Virasoro algebra. Cardy assumes the conjectured conformal invariance of percolation. (Aizenmann; Langlands, Pouliot, Saint-Aubin [LPSA94]).

Cardy's formula was for the crossing of a rectangle. Carleson observed that assuming conformal invariance it is equivalent to the above. Cardy's original formula involves hypergeometric functions.

Added in revision: For critical percolation on the triangular lattice Cardy's formula was recently proven by Stanislav Smirnov [Smi], who also showed that the scaling limit exists and is conformally invariant.

Langlands et al. attempted to understand Cardy's reasoning from a mathematical point of view, unsuccessfully, they say. However they presented the very appealing conformal invariance conjecture which they attribute to Aizenmann. This drew several mathematicians to this subject, myself included.

1.5. Uniform spanning trees (UST). Consider a random-uniform spanning tree of an $n \times n$ square in the grid $\mathbb{Z}^{2}$. 


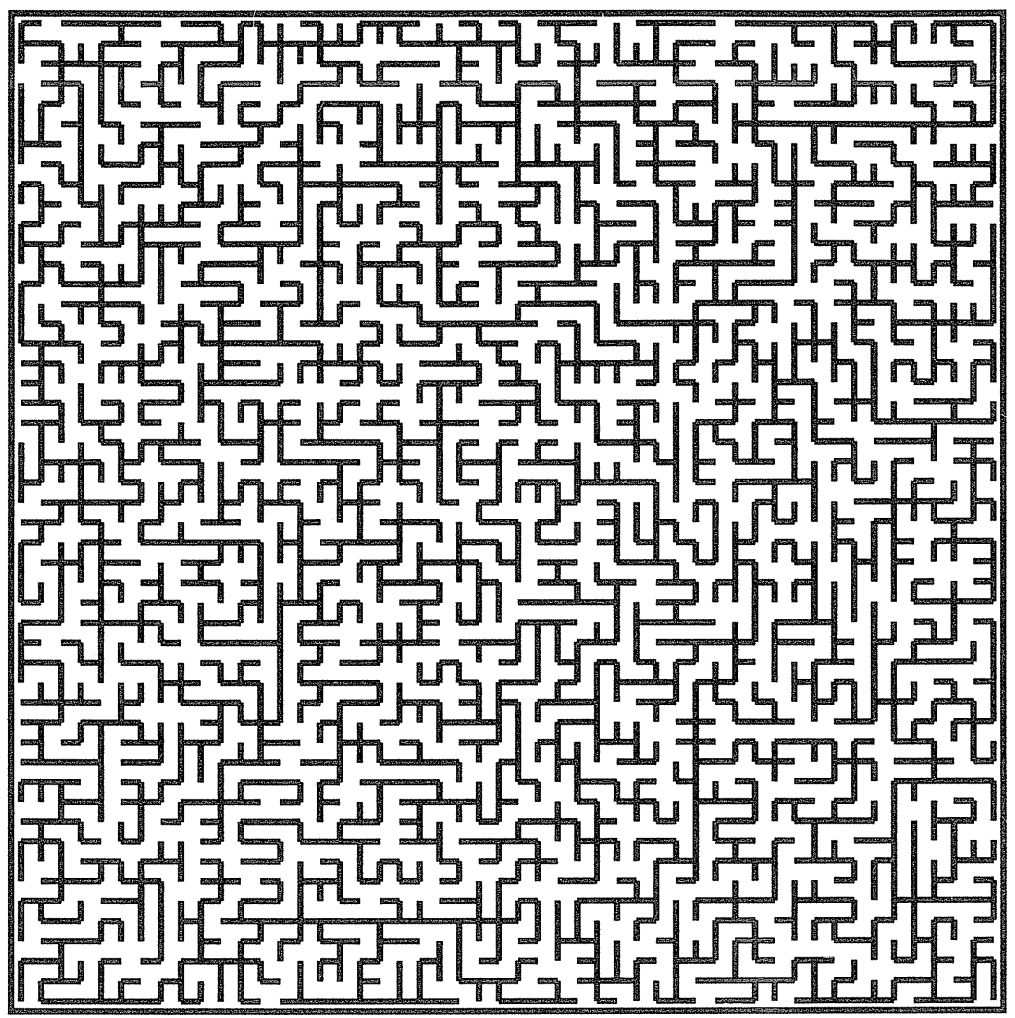

A spanning tree of a graph is a subgraph that contains all the vertices and where there is precisely one simple path joining any pair of distinct vertices.

A UST on a finite graph is a random spanning tree selected according to the uniform measure on the collection of all spanning trees of the graph. (That is, each spanning tree has the same probability of being selected.)

There is also an emerging theory of UST on infinite graphs, with contributions from Aizenman, Benjamini, Burchard, Häggström, Kenyon, Lyons, Newman, Pemantle, Peres, Schramm, Wilson and others.

One nice thing about the UST is that it connects well to classical mathematics, such as potential theory, and random walks.

1.6. Loop-erased random walk. If you fix two vertices $a, b$ in a finite graph $G$, then the UST path joining them is loop-erased random walk (LERW), from $a$ to $b$. 


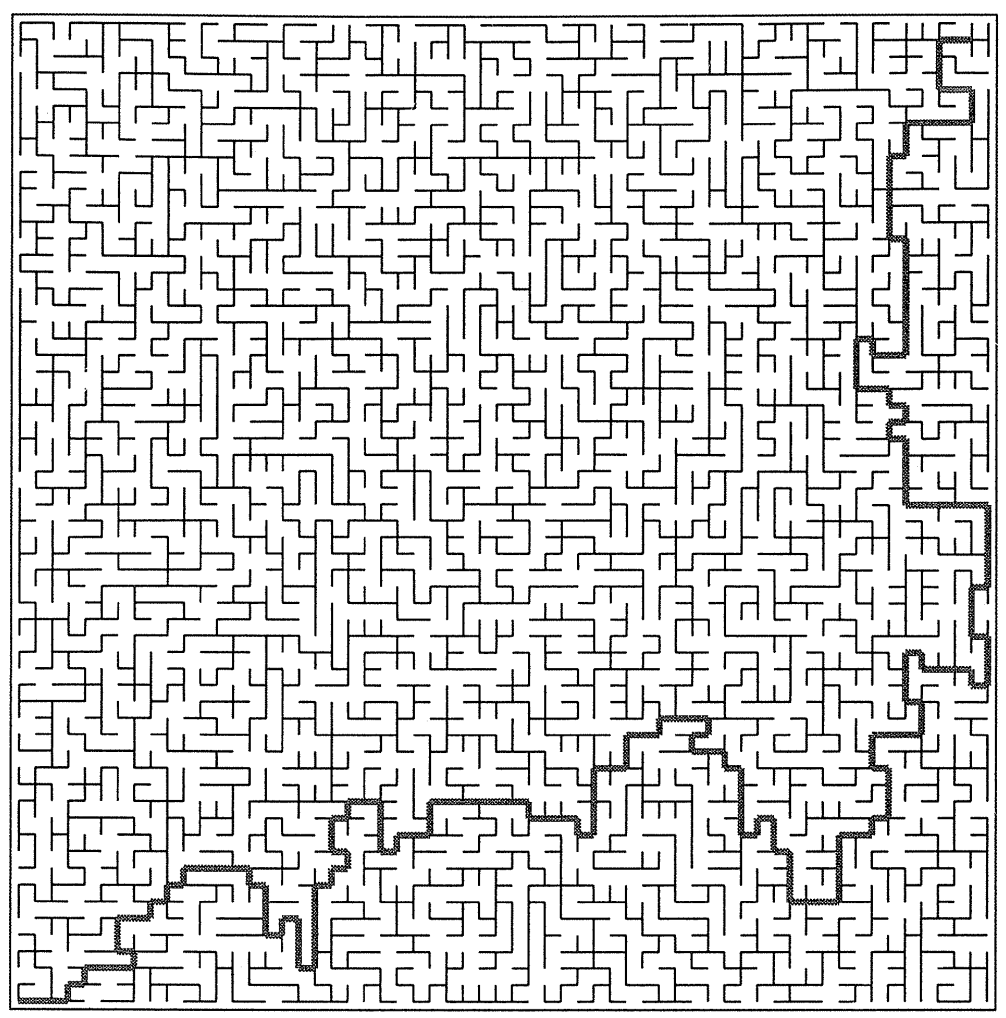

1.7. The definition of LERW. The LERW is obtained by performing simple random walk (SRW), and removing loops as they are created.

In other words, in the loop-erasure of a path $\gamma$, at each step you go from a vertex $v$ along the last edge of $\gamma$ incident with $v$. The notion of LERW was introduced by Greg Lawler [Law80]. The UST relation was first discovered by Aldous [Ald90] and Broder [Bro89].

Following is an algorithm due to Wilson [Wi196] for generating the UST of a finite graph $G$ using the LERW. Pick an arbitrary ordering $\left\{v_{1}, \ldots, v_{n}\right\}$ of the vertices of $G$. Inductively, we build a subtree $T_{j}$, as follows. Set $T_{1}=\left\{v_{1}\right\}$. Given $T_{j}, j<n$, let $L_{j+1}$ be the loop-erasure of the simple random walk started from $v_{j+1}$ which stops when it hits $T_{j}$. (If $v_{j+1} \in T_{j}$, then set $L_{j+1}=\left\{v_{j+1}\right\}$.) Set $T_{j+1}:=T_{j} \cup L_{j+1}$. Wilson proved that $T_{n}$ has the same distribution as the UST on $G$. 


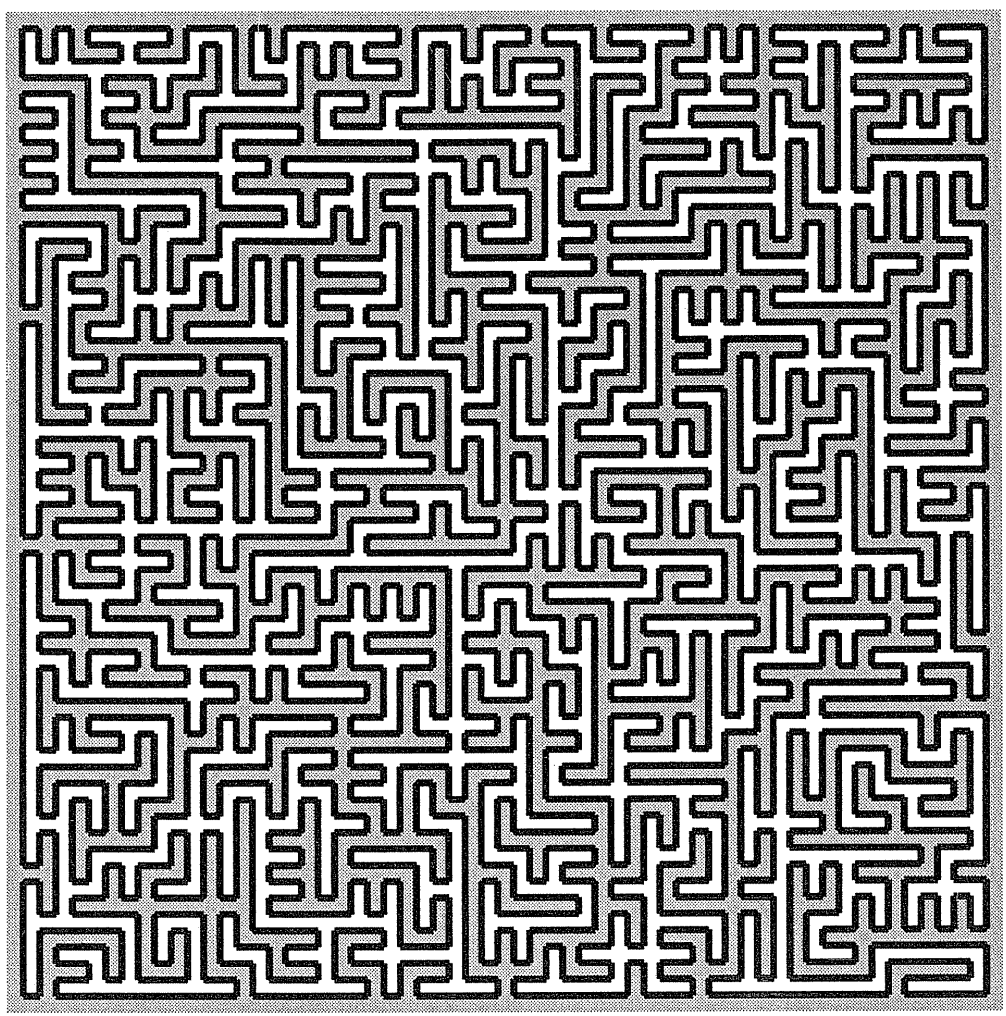

1.8. The Peano curve associated with the UST. The complement of the UST in the plane is another UST (on a dual grid). Between the UST and its dual winds the Peano path, which is shown in black in the figure.

Note that the UST Peano curve is space-filling, it visits every vertex in the appropriate grid.

1.9. The Big Conjecture. Conjecture. Percolation, UST, LERW and the Peano curve are conformally invariant in the scaling limit.

Special to 2 dimensions.

Added in revision: For percolation on the triangular grid, this has been recently established by Stanislav Smirnov [Smi].

In order to state this more precisely, one should define each of these processes in a domain. For example, for the LERW, let $D \subset \mathbb{R}^{2}$ be a domain and $p$ a basepoint in $D$. Take a fine square grid in the plane. Let $p^{\prime}$ be a vertex in the grid closest to $p$. Perform SRW on the grid starting from $p^{\prime}$ until the walk hits $\partial D$. Let $\gamma$ be the loop-erasure of this random walk. The conjectured conformal invariance applies to the (weak) limit of $\gamma$ as the mesh of the grid tends to zero, as a set of points, say, or as an unparameterized path.

Richard Kenyon [Ken00b, Ken00a, Ken00c, Ken] has shown that some properties of LERW and UST are conformally invariant in the scaling limit. His work is based on the relation with domino tilings.

There is much Monte-Carlo type and theoretical support for the conformal invariance conjecture. 
1.10. The Scaling limit of LERW. Consider the loop-erasure of a simple random walk starting at $a \in D$, which stops when $\partial D$ is first hit.

We may consider that as a random closed subset of $\bar{D}$.

The scaling limit is the limit of the law of this closed subset.

Theorem ([Sch00]). Any subsequential scaling limit of LERW is almost surely a simple path.

As we shall see below, there are natural situations where the scaling limit of a random simple path is not a simple path.

1.11. On the LERW scaling limit. Assuming the conformal-invariance of the LERW scaling limit, one can construct the limit directly. We now describe it.

Consider the LERW scaling limit $\gamma$ in the unit disk $\mathbb{U}$ starting from 0 . Consider a terminal segment $\gamma_{t}$ of the path $\gamma$ from some point $\gamma(t)$ to $\partial \mathbb{U}$. We may uniformize the complement of the segment in the unit disk, using Riemann's mapping theorem. That is, consider the conformal map $g_{t}: \mathbb{U} \backslash \gamma_{t} \rightarrow \mathbb{U}$ normalized by $g_{t}(0)=0$ and $g_{t}^{\prime}(0)>0$. This map is unique. The image of the tip $\gamma(t)$ under the conformal map $g_{t}$ is some point $\zeta(t)$ on the unit circle.

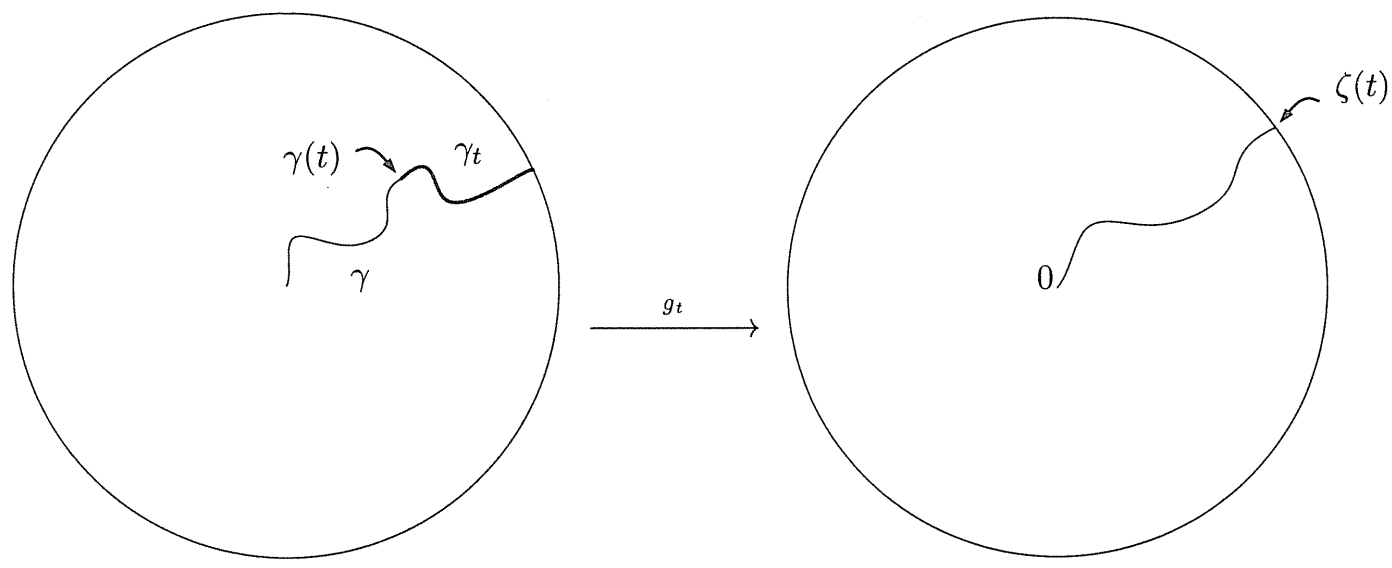

1.12. Theorem ([Sch00]). Assuming the conformal-invariance of LERW, $\zeta(t)$ is (1-dimensional) Brownian motion on the unit circle, run at twice the standard speed. (With the appropriate capacity parameterization of $\gamma$.)

The capacity parameterization of $\gamma$ is the unique parameterization $\gamma:[0, \infty] \rightarrow$ $\mathbb{U}$ such that $g_{t}^{\prime}(0)=\exp t$.

The theorem allows a reconstruction of $\gamma$, since by Löwner's theorem, $\gamma$ can be reconstructed from $\zeta$ :

Corollary ([Sch00]). Assuming that LERW has a conformally invariant scaling limit, the scaling limit of LERW from 0 to $\partial \mathbb{U}$ is the path

$$
\gamma(t)=f_{t}(\zeta(t)),
$$

where

$$
\zeta(t)=B(-2 t),
$$


$B(t)$ is $\mathrm{BM}$ on $\partial \mathbb{U}$, and $f_{t}$ is defined by Löwner's equation with parameter $\zeta$ :

$$
\frac{\partial}{\partial t} f_{t}(z)=z f_{t}^{\prime}(z) \frac{\zeta(t)+z}{\zeta(t)-z}, \quad f_{0}(z)=z .
$$

Remark: $f_{t}=g_{t}^{-1}$.

1.13. Sketch of the proof. The reasoning proceeds roughly as follows. LERW has the following combinatorial property (even before we pass to the scaling limit). If we condition on $\gamma_{t}$, the terminal part of the path from $\gamma(t)$ onward, the other arc, $\gamma \backslash \gamma_{t}$, is just LERW in the domain $D \backslash \gamma_{t}$ conditioned to hit the boundary at $\gamma(t)$. Using the assumed conformal invariance, we may then map $\mathbb{U} \backslash \gamma_{t}$ to the unit disk, and we are back at the start, except that the endpoint of the path is fixed. This identity of distribution gives the Markov property for $\zeta$, and also shows that $\zeta$ is stationary. It is then not hard to conclude that $\zeta$ is Brownian motion run at some constant speed.

To see that the speed is 2 , we calculate the asymptotics as $\epsilon \rightarrow 0$ of the variance of the winding number of the path around zero from the circle of radius $\epsilon$ to $\partial \mathbb{U}$, and compare with the analogous computation that Richard Kenyon [Ken00b] performed for LERW.

1.14. Other parameters? When $\zeta(t)=B(\kappa|t|)$, the solution of Löwner's differential equation with driving parameter $\zeta$ will be called Stochastic Löwner Evolution with parameter $\kappa$, or $S L E_{\kappa} . S L E_{2}$ (conjecturally) gives the scaling limit of LERW. What happens with other constants $\kappa$ ?

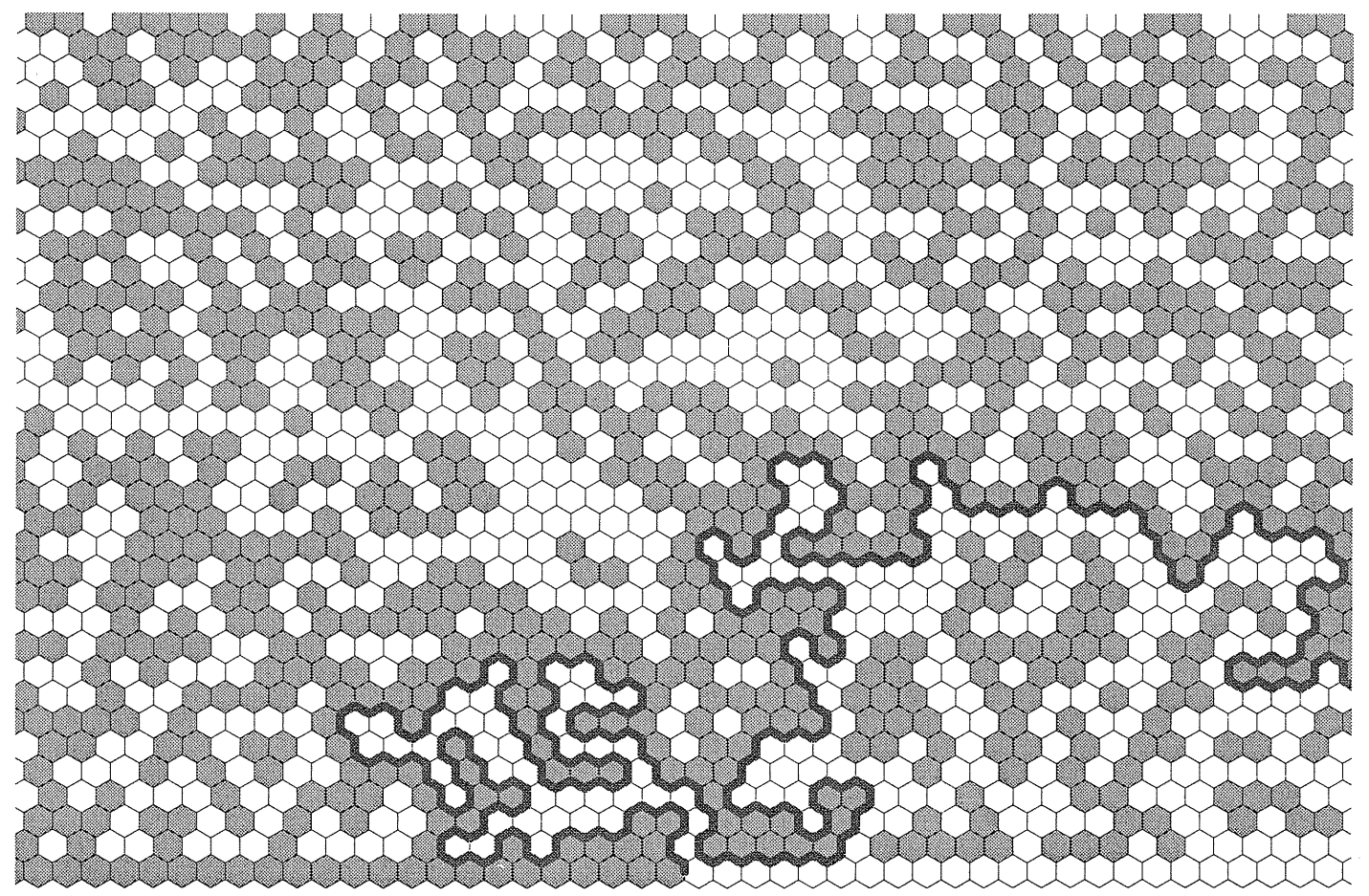


1.15. Critical percolation boundary path. In the figure, each of the hexagons is colored black with probability $1 / 2$, independently, except that the hexagons intersecting the positive real ray are all white, and the hexagons intersecting the negative real ray are all black. There is a boundary path $\beta$, passing through 0 and separating the black and the white connected components adjacent to 0 . The curve $\beta$ is a random path in the upper half-plane $\mathbb{H}$ connecting the boundary points 0 and $\infty$.

1.16. Critical percolation boundary path scaling limit. Theorem (S). Assuming the conformal invariance conjecture for critical percolation, the scaling limit of the path $\beta$ has the same law as $f_{t}(\zeta(t))$, where $\zeta(t)=B(6 t), B$ is Brownian motion in $\mathbb{R}$ starting at $B(0)=0$, and $f_{t}$ is the solution of a Löwner-like equation with parameter $\zeta$ :

$$
\frac{\partial}{\partial t} f_{t}(z)=\frac{2 f_{t}^{\prime}(z)}{\zeta(t)-z}
$$

with $f_{0}(z)=z$.

Remark. It can be shown that the scaling limit of the percolation boundary curve is not a simple path.

1.17. Two versions of SLE. Note that the LERW path is a path from an interior point in the domain to a boundary point, while the percolation boundary curve is a path joining two endpoints of the domain ( 0 and $\infty$, in this case).

This requires somewhat different treatment. The normalization of the conformal map changes, and hence the differential equation changes too.

The version where two points on the boundary are joined is called chordal SLE while the version where a point in the interior is joined to the boundary is called radial SLE.

It turns out that radial and chordal SLE are essentially the same.

1.18. UST Peano. At $\kappa=2$, we "got" LERW. At $\kappa=6$, we "got" percolation. Assuming that LERW has a conformally invariant scaling limit, it follows that chordal $S L E_{8}$ is the scaling limit of the UST Peano curve (with partially wired boundary). 


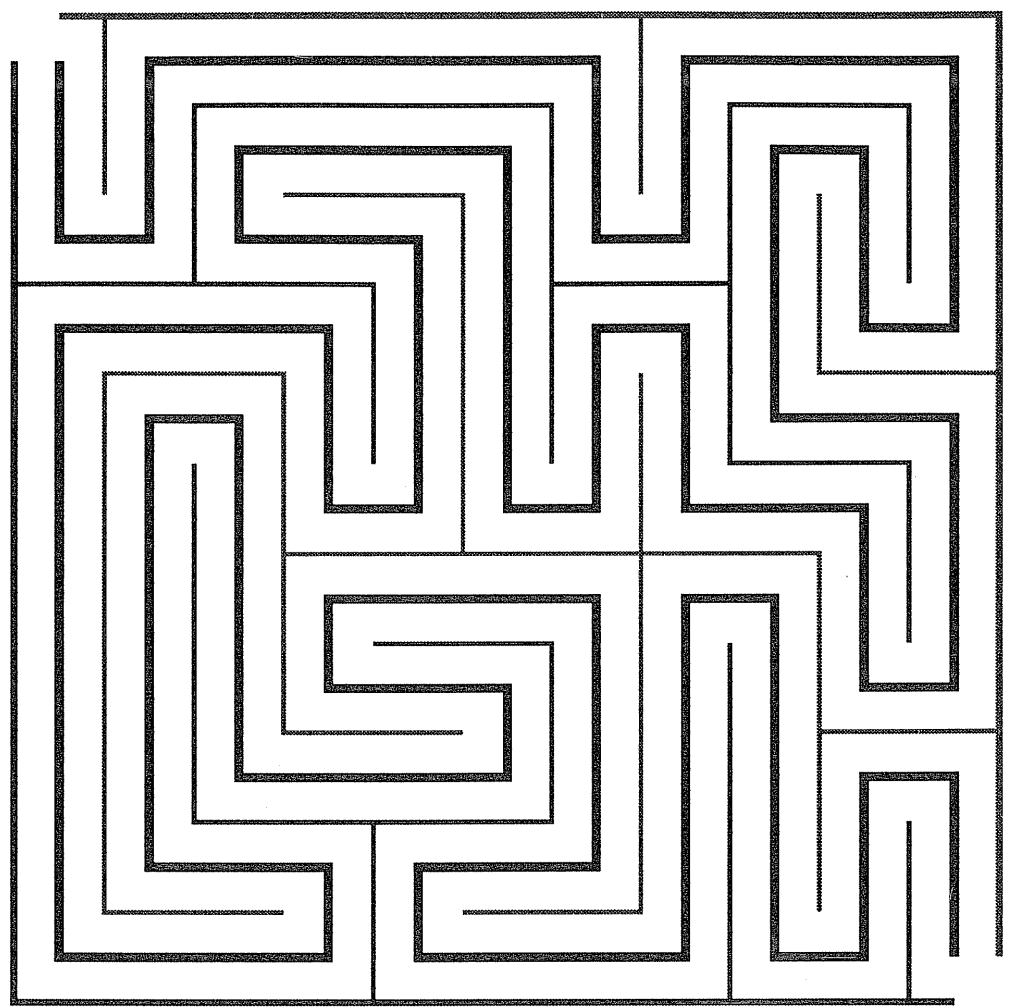

In the figure, the square boundary is wired on the lower and left edges. The dual graph is then wired on the right and top edges. The Peano curve then joins the lower right and the upper left corners.

1.19. Phases of SLE. Theorem (Rohde-Schramm $[\mathbf{R S}]$ ). For all $\kappa \geqslant 0$, $\kappa \neq 8$, SLE produces a continuous path. It is a simple path iff $\kappa \leqslant 4$. It is space filling iff $\kappa>8$.

The SLE path is defined by $f_{t}(\zeta(t))$. Continuity is nontrivial, since it is not a priori clear that $f_{t}$ extends continuously to the boundary.

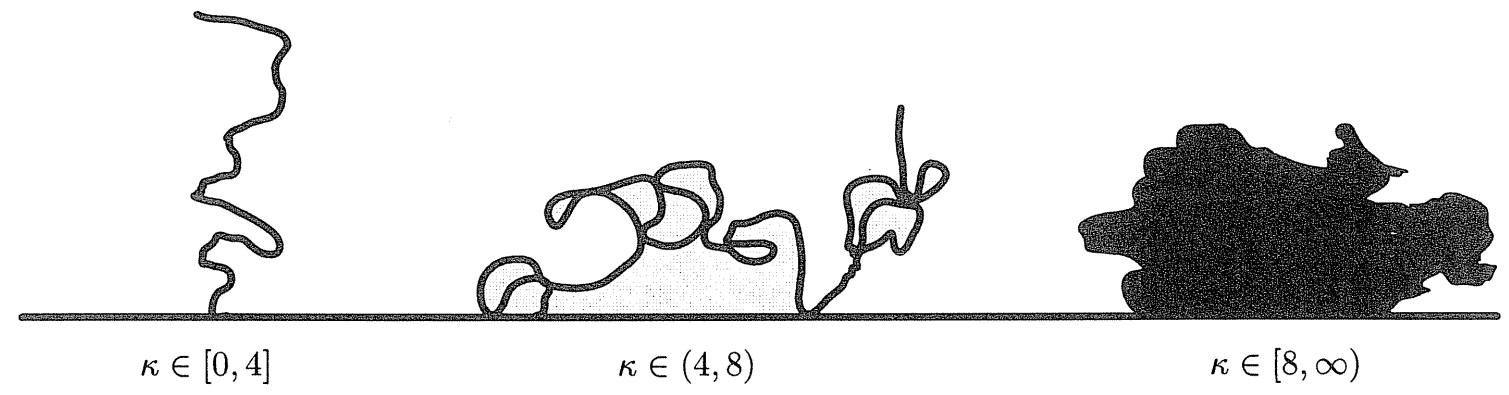

In the phase $\kappa \in(4,8)$, the SLE path makes loops "swallowing" parts of the domain. However, it never crosses itself. 
The Hausdorff dimension of the SLE path is conjectured to be $1+\kappa / 8$ when $\kappa \leqslant 8$.

1.20. Computing with SLE. One can compute using the SLE. Typically, problems about SLE convert to PDE questions.

Cardy's formula has been proven for $\mathrm{SLE}_{6}$ (Lawler-Schramm-Werner [LSW1]). This gives a proof of Cardy's formula modulo the conformal invariance conjecture.

Also, various generalizations and variations on Cardy's formula have been proven for SLE.

1.21. Example calculation sketch: Cardy's formula. Recalling the definition of the percolation boundary path, it is clear what Cardy's formula for SLE should be. It is a formula describing the probability that $I:=(-\infty,-1)$ is hit by the SLE before $J:=(s, \infty)$, as a function of $s, s>0$. (Conformal invariance is used here.)

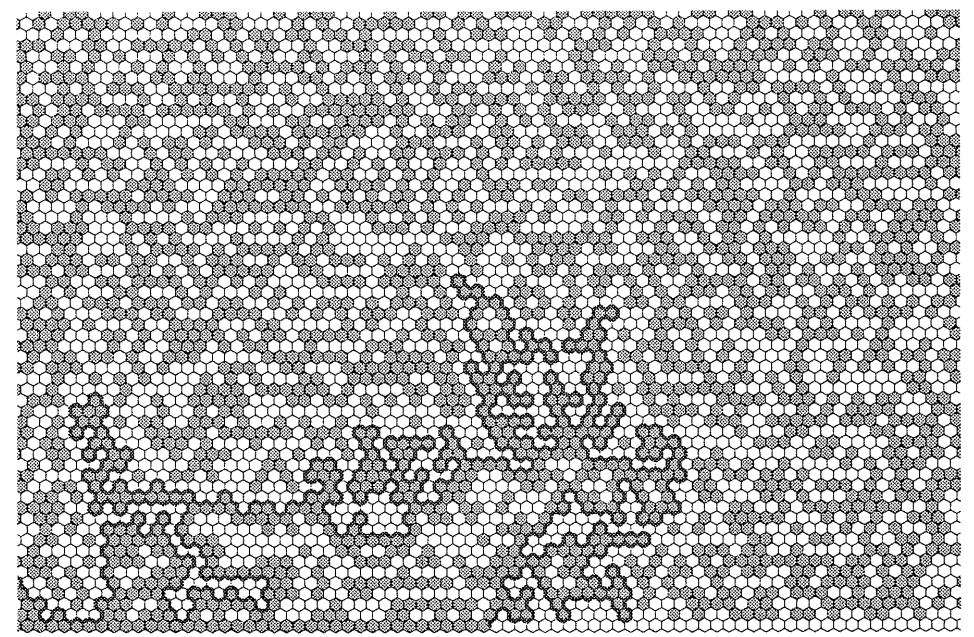

The differential equation for the (normalized) map going from the half-plane slitted by the SLE to the half-plane is

$$
\partial_{t} g_{t}(z)=\frac{2}{g_{t}(z)-\zeta(t)} .
$$

We run this random flow from $g_{0}(z)=z$ and look for the probability that $g_{t}(s)$ hits $\zeta(t)$ before $g_{t}(-1)$ does.

This is so because when $t$ increases to the time when $s$ is "swallowed" by the SLE, $g_{t}(s)-\zeta(t) \rightarrow 0$. This is a little exercise in conformal maps.

Note that one only needs to keep track of $g_{t}(s), g_{t}(-1)$ and $\zeta(t)$, the behaviour of $g$ at other points can be ignored.

Let $F(a, b)$ be the probability that in this flow $g_{t}(a)$ hits $\zeta(t)$ before $g_{t}(b)$, where $\zeta(0)=0$. It is clear that $F\left(g_{t}(a)-\zeta(t), g_{t}(b)-\zeta(t)\right)$ is a (local) martingale. This gives a PDE for $F$. Moreover, scale invariance shows that $F(\lambda a, \lambda b)=F(a, b)$ for $\lambda>0$. This reduces the PDE to an ODE. Cardy's formula in the upper half plane is the solution of the ODE. 
1.22. $\mathrm{SLE}_{6}$ and the outer boundary of planar BM. There is a version of SLE in the whole plane started from 0 and going to $\infty$, which we have not discussed. Call it full-plane SLE.

Theorem (Lawler-Schramm-Werner). Let $F$ be the outer boundary of planar BM started from 0 and stopped on hitting the unit circle. Let $F^{\prime}$ be the outer boundary of full plane $\mathrm{SLE}_{6}$ stopped on hitting the unit circle. Then $F$ and $F^{\prime}$ have the same distribution.

The outer boundary of a closed bounded set $X \subset \mathbb{R}^{2}$ is its intersection with the closure of the unbounded connected component of the complement of $X$.

Note: there is no conjecture assumed, because conformal invariance is known for $\mathrm{BM}$.

1.23. Summary. $S L E_{\kappa}$ is conjectured to be the scaling limit of LERW at $\kappa=2$, of the percolation boundary path at $\kappa=6$, and of the UST Peano path at $\kappa=8$. There are also conjectures for $\kappa=4$ and for all $\kappa \in[0,8]$.

The outer boundary of $\mathrm{SLE}_{6}$ is essentially identical with the outer boundary of planar BM.

The study of properties of $S L E_{\kappa}$ is in progress.

\section{Applications to planar Brownian motion}

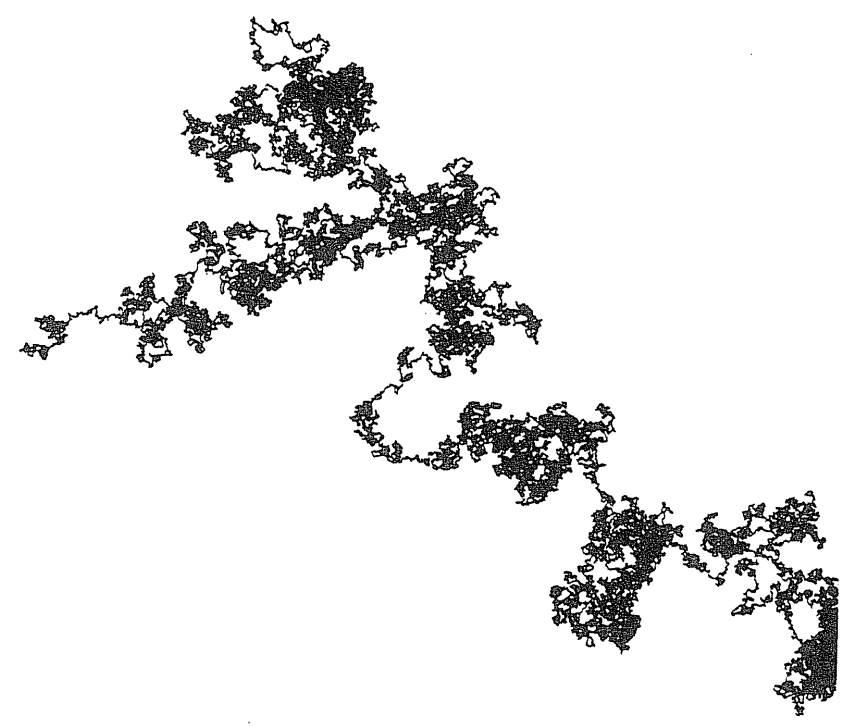

2.1. What is Brownian motion? Define simple random walk on $\mathbb{Z}$ starting at 0 by setting $X(0)=0$ and for each $n \in \mathbb{N}$, given $X(1), \ldots, X(n)$, let $X(n+1)$ be $X(n)-1$ or $X(n)+1$, each with probability $1 / 2$. For $t \in[n, n+1)$ set $X(t)=X(n)$.

$\mathrm{BM}$ on $\mathbb{R}$ is the limit as $\delta \rightarrow 0$ of the process $t \mapsto \delta X\left(t / \delta^{2}\right)$.

It has amazing properties. For one, if $B_{1}$ and $B_{2}$ are independent BM's starting at 0 , then $\left(B_{1}, B_{2}\right)$ is rotationally invariant. It is called 2-dimensional BM. 
2.2. Conformal invariance of $\mathbf{B M}$. In fact, 2-dimensional BM is also conformally invariant if we ignore the time parameterization. That is, suppose that $f: D \rightarrow D^{\prime}$ is a conformal homeomorphism between domains in $\mathbb{R}^{2}$ and $f(0)=0$. Let $B$ be BM starting from zero stopped when it hits $\partial D$, and let $B^{\prime}$ be BM starting from zero stopped when it hits $\partial D^{\prime}$. Then $f(B)$ and $B^{\prime}$ have the same distribution, as unparameterized paths.

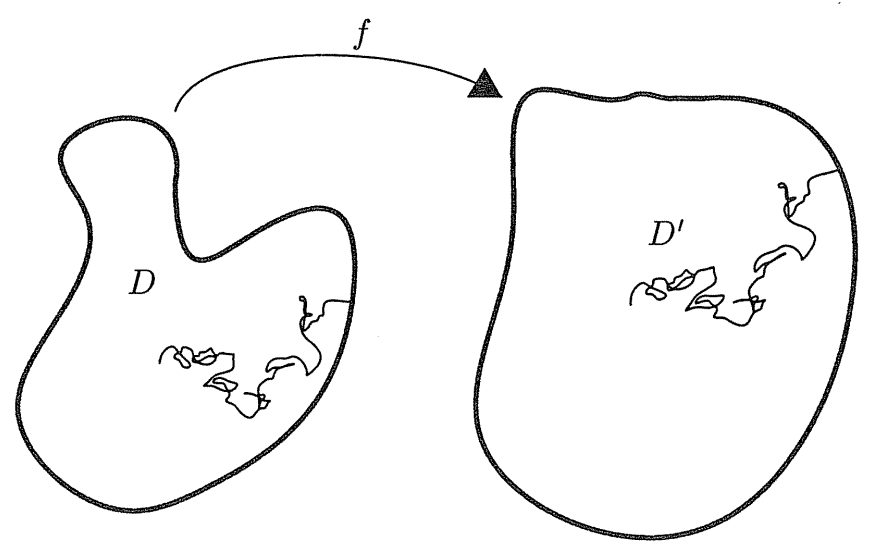

2.3. Brownian intersection exponents. Consider BM in the plane. The simplest BM exponent is $\xi(1,1)$ :

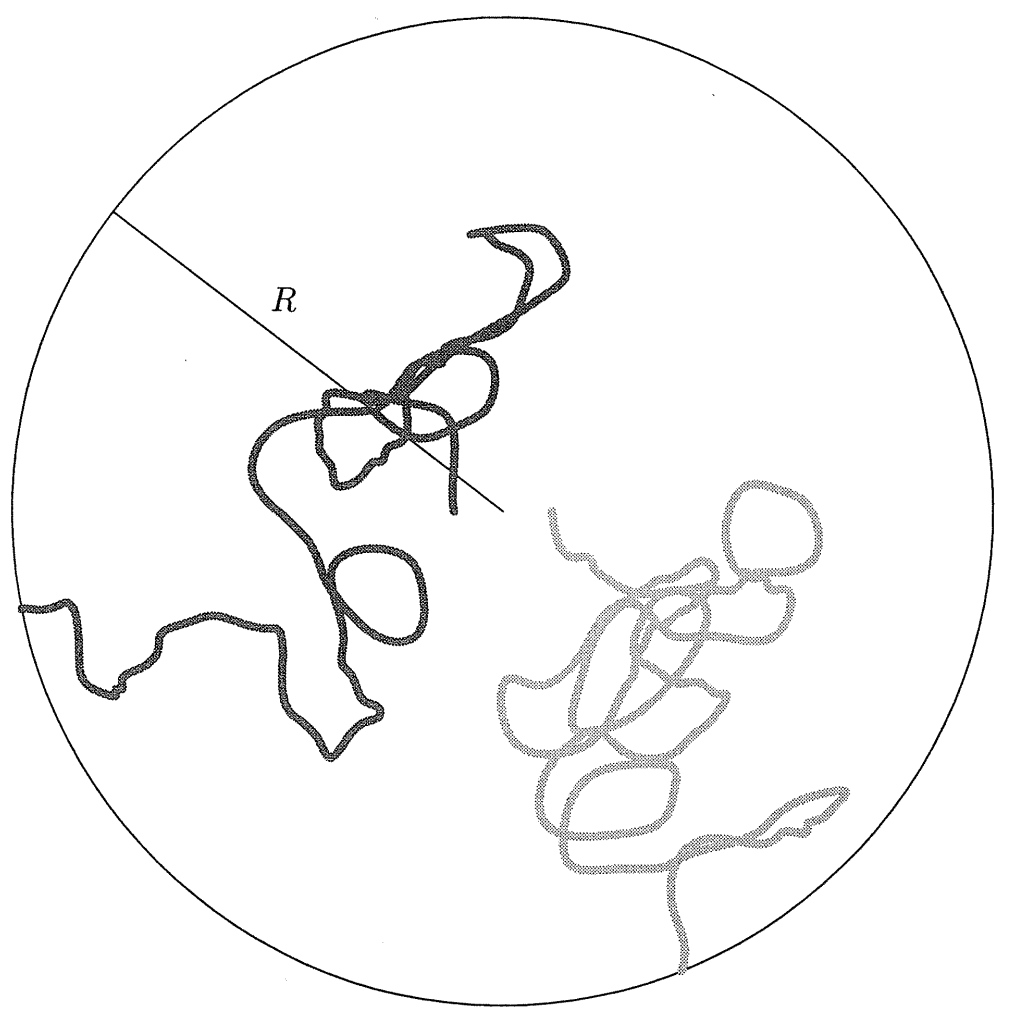


Run two brownian motions in the plane, "green" $B$ starts from 1 , and "red" $B$ " starts from -1 . Consider the probability that the paths do not intersect until they hit the circle of radius $R$ about 0 . It is not hard to see that this probability decays like a power of $R$ as $R \rightarrow \infty$. Thus, the intersection exponent $\xi(1,1)$ is defined by

$$
\mathbf{P}\left[\mathbf{B} \cap \mathbf{B}^{\prime}=\emptyset\right]=\mathbf{R}^{-\xi(\mathbf{1}, \mathbf{1})+\mathbf{o}(\mathbf{1})}, \quad \mathbf{R} \rightarrow \infty .
$$

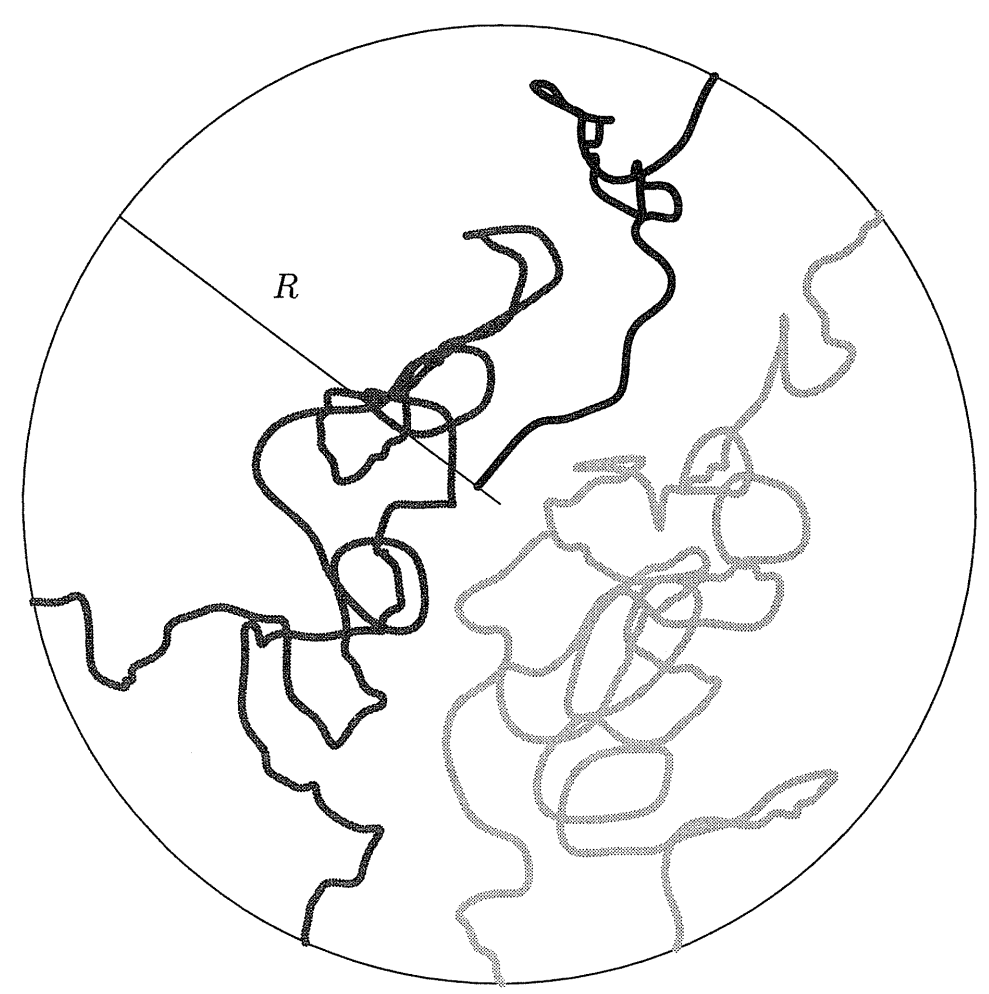

\subsection{More general exponents.}

$$
P \text { [different colors stay disjoint }]=R^{-\xi(2,1,3)+o(1)}
$$

Similarly, other intersection exponents can be defined. For example, suppose you had two independent red BM's, one blue BM and 3 green BM, then the probability that paths of different color do not intersect until they hit the circle of radius $R$ decays like $R^{-\xi(2,1,3)+o(1)}$.

2.5. Significance of the exponents. The exponents encode much information about BM and SRW. For example, the probability that two SRW paths of $n$ steps each starting from zero will not intersect again decays like $n^{-\xi(1,1) / 2}$ (BurdzyLawler [BL90]).

The Hausdorff dimension of the set of cut points of $B[0,1]$ is almost surely $2-\xi(1,1)$ (Lawler [Law96b]).

A cut point of a connected set $X$ is a point $p \in X$ such that $X \backslash\{p\}$ is disconnected.

2.6. Determination of the exponents. The values of the exponents $\xi(1,1, \ldots, 1)$ have been conjectured by Duplantier-Kwon [DK88]. The following theorem proves a generalization of this conjecture. 


\section{Theorem (Lawler-Schramm-Werner [LSW3]).}

$$
\begin{aligned}
& \xi\left(n_{1}, n_{2}, \ldots, n_{k}\right)= \\
& \quad \frac{\left(\sqrt{24 n_{1}+1}+\cdots+\sqrt{24 n_{k}+1}-k\right)^{2}-4}{48} .
\end{aligned}
$$

Corollary [LSW2]. The Hausdorff dimension of the set of cut points of $B[0,1]$ is $3 / 4$ almost surely.

2.7. Heuristic. Here's an imprecise explanation for the relation between the dimension of the set of cut points and the exponent $\xi(1,1)$.

In order for $p$ to be a cut point, it first has to lie on the path. But the probability that in a disk of radius $\epsilon$ there will be a point on the path decays slowly (logarithmically) as $\epsilon \rightarrow 0$.

Given that $p$ is on the path, in order for it to be a cut point we need the "past" (the part before hitting $p$ ) and the "future" (the part after hitting $p$ ) to be disjoint. By reversability of BM, the "probability" for that is essentially the same as the probability that two independent BM's starting from $p$ will not intersect. The decay for this event is governed by $\xi(1,1)$.

The 2 in the formula $2-\xi(1,1)$ comes from the 2 -dimensionality of $\mathbb{R}^{2}$. The $-\xi(1,1)$ is measures the improbability that two independent BM's will not intersect.

The proof follows along these lines.

2.8. Disconnection exponents. Let $f_{k}(R)$ be the probability that $k$ independent BM paths in $\mathbb{R}^{2}$ starting from 1 will hit the circle of radius $R$ about 0 before disconnecting 0 from $\infty$. The disconnection exponent $\eta(k)$ is defined by the relation

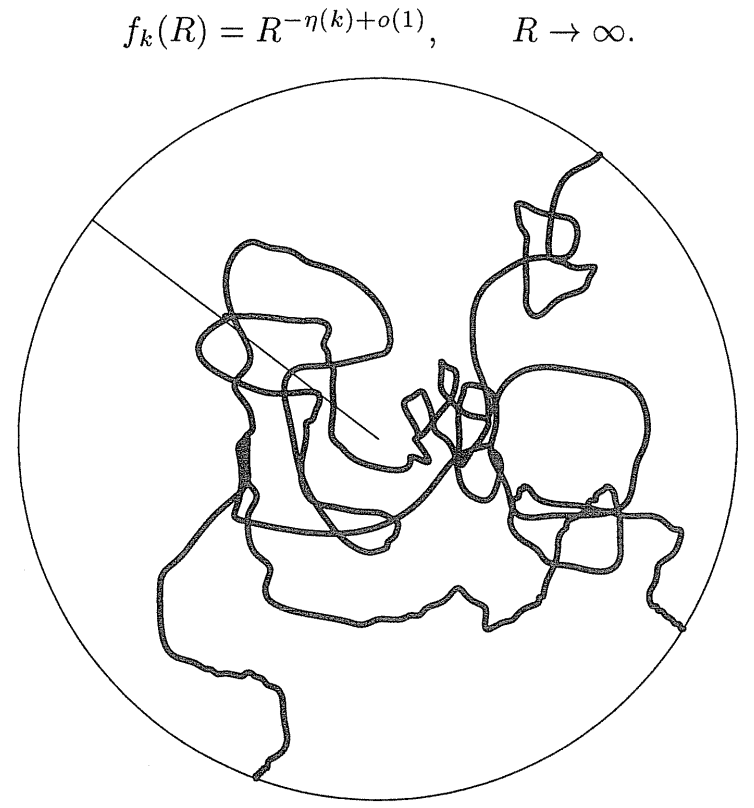

The event defining $\eta(3)$. 
Theorem (Lawler-Schramm-Werner [LSW4]).

$$
\eta(k)=\frac{(\sqrt{24 k+1}-1)^{2}-4}{48} .
$$

2.9. Brownian frontier. Corollary (Lawler-Schramm-Werner [LSW4]). The Hausdorff dimension of the outer boundary of $B[0,1]$ is almost surely $4 / 3$. (As conjectured by Mandelbrot.)

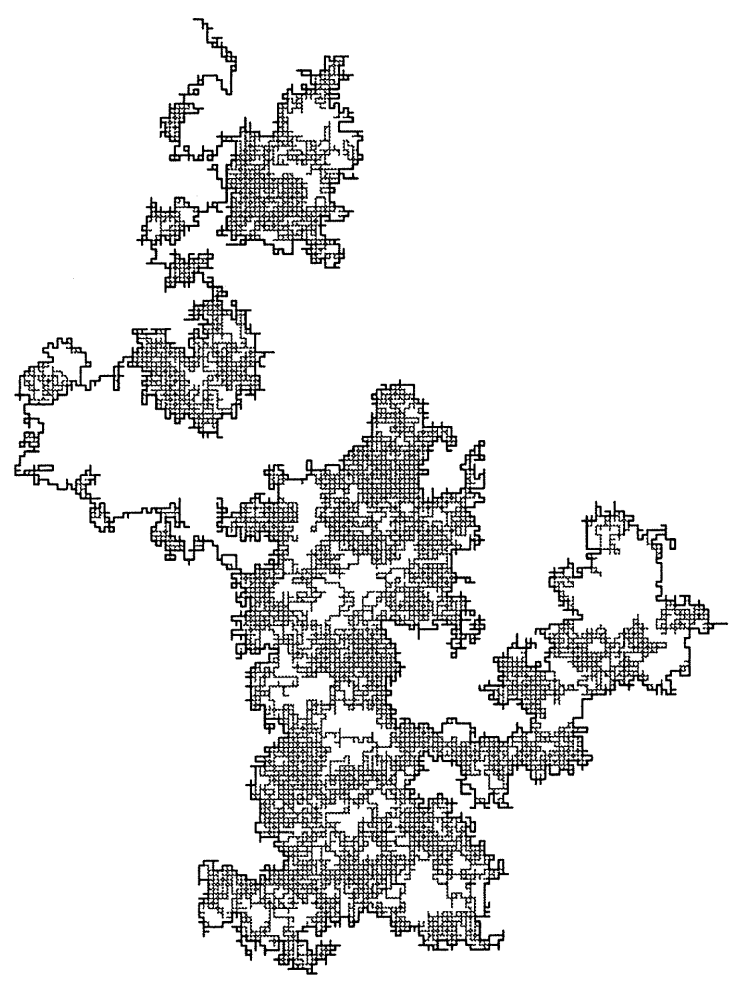

Proof: This follows from $\eta(2)=2 / 3$ and a result of Lawler [Law96a] showing that the hausdorff dimension of the outer boundary is equal to $2-\eta(2)$.

Exercise: Figure out the heuristic for the result of Lawler that $2-\eta(2)$ should be the dimension of the outer boundary of planar BM.

2.10. Universality. Suppose that to every simply connected domain $D \subset \mathbb{C}$, we have a random set $X=X_{D}$ in the closure $\bar{D}$. $X$ may also depend on one or two points in the interior or boundary of $D$. (Call those anchor points.) E.g., BM, LERW, or percolation boundary curve.

Lawler and Werner [LW99, LW00] showed that if the process $X$ satisfies

- conformal invariance,

- locality, and

- other stuff,

then the intersection exponents of $B M$ can be determined from those of $X$. This they called universality. 
Conformal invariance means that when $f: D \rightarrow D^{\prime}$ is conformal, the distribution of $f\left(X_{D}\right)$ is the same as that of $X_{D^{\prime}}$, with the anchor points mapped by $f$.

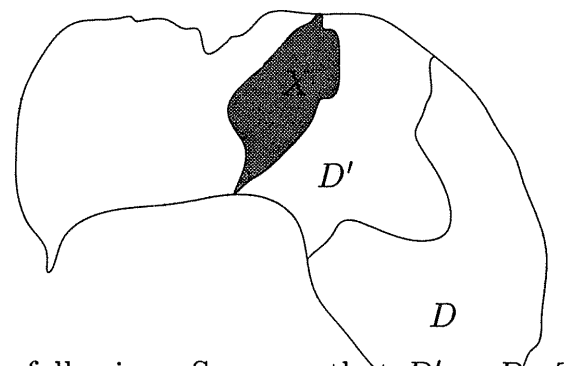

Locality means the following. Suppose that $D^{\prime} \subset D$. Then the restriction of $X_{D^{\prime}}$ to the event $X_{D^{\prime}} \cap \partial D^{\prime} \cap D=\emptyset$ has the same distribution as the restriction of $X_{D}$ to the event $X_{D} \cap \partial D^{\prime} \cap D=\emptyset$.

2.11. The universality argument (LW). Suppose that $X$ has a single anchor point $p$ in the interior of the domain $D$, and $X$ is a random set containing $p$ and having a single point on the boundary $\partial D$.

Then conformal invariance implies that (when $D$ is simply connected) $X \cap \partial D$ has the distribution of harmonic measure on $\partial D$. That is, it is the pull-back of uniform measure on the unit circle under the Riemann map from $D$ to $\mathbb{U}$ taking $p$ to 0 .

Let $B$ and $B^{\prime}$ be two independent BM's starting from $p$ and $q$, respectively, in $R \mathbb{U}$. Let $X$ start from $q$.

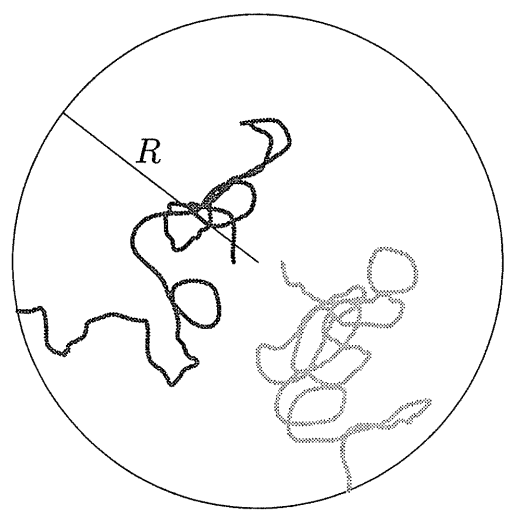

For a set $Y \subset R \overline{\mathbb{U}}$, let $h_{q}(Y)$ denote the harmonic measure from $q$ of $Y$ in $R \mathbb{U} \backslash Y$.

The following shows that one may replace $B^{\prime}$ with $X$ when calculating the non-intersection probability.

$$
\begin{aligned}
P\left[B \cap B^{\prime}=\emptyset\right] & =E\left[P\left[B \cap B^{\prime}=\emptyset \mid B\right]\right] \\
& =E\left[1-h_{q}(B)\right] \\
& =E[P[B \cap X=\emptyset \mid B]] \\
& =P[B \cap X=\emptyset] .
\end{aligned}
$$


In the second and last equalities, locality and conformal invariance for $B^{\prime}$ and $X$ are used.

2.12. How SLE fits in. At roughly the same time, I defined $S L E$ [Sch00] and conjectured that $\mathrm{SLE}_{6}$ is the scaling limit of the percolation boundary curve.

$S L E_{\kappa}$ can be defined in an arbitrary simply connected domain via conformal maps. It is then conformally invariant.

Assuming that $\mathrm{SLE}_{6}$ is the scaling limit of the percolation boundary path, locality is obvious. Without the conjecture, locality was established [LSW1] with difficulty. The "other stuff" got handled differently.

Universality allows to replace the calculation of the exponents for BM with the exponents for $\mathrm{SLE}_{6}$.

2.13. Exponents calculation for $\mathrm{SLE}_{6}$. As the above universality calculation shows, it suffices to be able to determine the distribution of $h_{0}\left(\mathrm{SLE}_{6}\right)$. That is, in the SLE evolution, we need to keep track of the length of the image $g_{t}(\partial \mathbb{U})$. It turns out that it suffices to consider instead the decay of $\left|g_{t}^{\prime}(1)\right|$. This can be converted to a PDE problem, since the infinitesimal evolution is known.

Why are exponents easier for $\mathrm{SLE}_{6}$ ?

- Since $\mathrm{SLE}_{6}$ does not cross itself, we only need to keep track of the exterior of the domain. By conformal invariance, this reduces to a (small) finite number of parameters.

- Reduction of the problem to 1-dimension (BM on $\mathbb{R})$.

2.14. When universality fails. The above is an outline for the calculation of the $\xi\left(n_{1}, \ldots, n_{k}\right)$ exponents. However, the universality argument does not seem to work for the disconnection exponents.

2.15. Generalized exponents. The exponent $\xi(k, \lambda)$ can be naturally defined for $\lambda \geqslant 0$ which is not necessarily an integer, as follows.

Let $B_{1}, \ldots, B_{k}$ be $k$ independent BM's starting from $1 \in \mathbb{C}$ and stopped when they hit $R \partial \mathbb{U}$, and let $B$ be an independent $B M$ from 0 , which is also stopped when it hits $R \partial \mathbb{U}$.

Given $B_{1}, \ldots, B_{k}$, let $Z=Z\left(B_{1}, \ldots, B_{k}\right)$ be the probability that $B$ does not intersect $B_{1} \cup \cdots \cup B_{k}$ :

$$
Z=\mathbb{P}\left[B \cap\left(B_{1} \cup \cdots \cup B_{k}\right)=\emptyset \mid B_{1}, \ldots, B_{k}\right] .
$$

In other words, $Z$ is the harmonic measure from 0 of $R \partial \mathbb{U}$ in $R \mathbb{U} \backslash\left(B_{1} \cup \cdots \cup B_{k}\right)$.

Then $\xi(k, \lambda)$ is defined by the relation

$$
\mathbb{E}\left[Z^{\lambda}\right]=R^{-\xi(k, \lambda)+o(1)}, \quad R \rightarrow \infty .
$$

Note that this coincides with the previous definition when $\lambda=1,2, \ldots$.

2.16. Analyticity. Lawler [Law00] showed that $\eta(k)=\lim _{\lambda \searrow 0} \xi(k, \lambda)$.

The determination of the disconnection exponents $\eta(k)$ then proceeds by first determining $\xi(k, \lambda)$ when $\lambda$ is large. In this range, universality can be used. Then we $[\mathbf{L S W} 4]$ show that $\xi(k, \lambda)$ is an analytic function of $\lambda$. Hence, one gets the formula for $\eta(k)$ by analytic continuation.

There's hope for a more direct proof. 


\section{References}

[AB99] M. Aizenman and A. Burchard. Hölder regularity and dimension bounds for random curves. Duke Math. J., 99(3):419-453, 1999.

[ABNW99] Michael Aizenman, Almut Burchard, Charles M. Newman, and David B. Wilson. Scaling limits for minimal and random spanning trees in two dimensions. Random Structures Algorithms, 15(3-4):319-367, 1999. Statistical physics methods in discrete probability, combinatorics, and theoretical computer science (Princeton, NJ, 1997).

[Ald90] David J. Aldous. The random walk construction of uniform spanning trees and uniform labelled trees. SIAM J. Discrete Math., 3(4):450-465, 1990.

[BL90] Krzysztof Burdzy and Gregory F. Lawler. Nonintersection exponents for Brownian paths. II. Estimates and applications to a random fractal. Ann. Probab., 18(3):981$1009,1990$.

[BLPS01] I. Benjamini, R. Lyons, Y. Peres, and O. Schramm. Uniform spanning forests. Ann. Probab., 29:1-65, 2001.

[Bro89] Andrei Broder. Generating random spanning trees. In Foundations of Computer Science, pages 442-447, 1989.

[Car92] John L. Cardy. Critical percolation in finite geometries. J. Phys. A, 25(4):L201-L206, 1992.

[DK88] Bertrand Duplantier and K.-H. Kwon. Conformal invariance and intersection of random walks. Phys. Rev. Let., pages 2514-2517, 1988.

[Gri89] Geoffrey Grimmett. Percolation. Springer-Verlag, New York, 1989.

[Häg95] Olle Häggström. Random-cluster measures and uniform spanning trees. Stochastic Process. Appl., 59(2):267-275, 1995.

[Häg98] Olle Häggström. Uniform and minimal essential spanning forests on trees. Random Structures Algorithms, 12(1):27-50, 1998.

[Har60] T. E. Harris. A lower bound for the critical probability in a certain percolation process. Proc. Cambridge Philos. Soc., 56:13-20, 1960.

[HS90] Takashi Hara and Gordon Slade. Mean-field critical behaviour for percolation in high dimensions. Comm. Math. Phys., 128(2):333-391, 1990.

[Ken00a] Richard Kenyon. Long-range properties of spanning trees. J. Math. Phys., 41(3):13381363, 2000. Probabilistic techniques in equilibrium and nonequilibrium statistical physics.

[Ken00b] Richard Kenyon. Conformal invariance of domino tiling. Ann. Probab., 28(2):759-795, 2000 .

[Ken00c] Richard Kenyon. The asymptotic determinant of the discrete laplacian. Acta Math., 185(2):239-286, 2000.

[Ken] Richard Kenyon. Dominos and the Gaussian free field, arXiv:math-ph/0002027.

[Kes80] Harry Kesten. The critical probability of bond percolation on the square lattice equals $\frac{1}{2}$. Comm. Math. Phys., 74(1):41-59, 1980.

[Law80] Gregory F. Lawler. A self-avoiding random walk. Duke Math. J., 47(3):655-693, 1980.

[Law96a] G. Lawler. The dimension of the frontier of planar Brownian motion. Electron. Comm. Probab., 1:no. 5, 29-47, 1996.

[Law96b] Gregory F. Lawler. Hausdorff dimension of cut points for Brownian motion. Electron. J. Probab., 1:no. 2, approx. 20 pp., 1996.

[Law00] Gregory F. Lawler. Strict concavity of the half plane intersection exponent for planar Brownian motion. Electron. J. Probab., 5:no. 8, 33 pp., 2000.

[LPSA94] Robert Langlands, Philippe Pouliot, and Yvan Saint-Aubin. Conformal invariance in two-dimensional percolation. Bull. Amer. Math. Soc. (N.S.), 30(1):1-61, 1994.

[LSW1] Gregory F. Lawler, Oded Schramm, and Wendelin Werner. Values of Brownian intersection exponents I: Half-plane exponents. Acta Math., to appear. arXiv:math.PR/9911084.

[LSW2] Gregory F. Lawler, Oded Schramm, and Wendelin Werner. Values of Brownian intersection exponents II: Plane exponents. Acta Math., to appear. arXiv:math.PR/0003156.

[LSW3] Gregory F. Lawler, Oded Schramm, and Wendelin Werner. Values of Brownian intersection exponents III: Two-sided exponents. Acta Math., to appear. arXiv:math.PR/0005294. 
[LSW4] Gregory F. Lawler, Oded Schramm, and Wendelin Werner. Analyticity of intersection exponents for planar Brownian motion, Acta Math., to appear. arXiv:math.PR/0005295.

[LSW5] Gregory F. Lawler, Oded Schramm, and Wendelin Werner. The dimension of the planar Brownian frontier is 4/3. Math. Res. Lett., to appear.

[LW99] Gregory F. Lawler and Wendelin Werner. Intersection exponents for planar Brownian motion. Ann. Probab., 27(4):1601-1642, 1999.

[LW00] Gregory F. Lawler and Wendelin Werner. Universality for conformally invariant intersection exponents. J. Eur. Math. Soc. (JEMS), 2(4):291-328, 2000.

[Pem91] Robin Pemantle. Choosing a spanning tree for the integer lattice uniformly. Ann. Probab., 19(4):1559-1574, 1991.

[RS] Steffen Rohde and Oded Schramm. Basic properties of SLE. arXiv:math.PR/0106036.

[Sch00] Oded Schramm. Scaling limits of loop-erased random walks and uniform spanning trees. Israel J. Math., 118:221-288, 2000.

[Smi] Stanislav Smirnov. Critical percolation in the plane. I. Conformal invariance and Cardy's formula. II. Continuum scaling limit. Preprint.

[Wer00] Wendelin Werner. Critical exponents, conformal invariance and planar Brownian motion, 2000, arXiv:math.PR/0007042.

[Wil96] David Bruce Wilson. Generating random spanning trees more quickly than the cover time. In Proceedings of the Twenty-eighth Annual ACM Symposium on the Theory of Computing (Philadelphia, PA, 1996), pages 296-303, New York, 1996. ACM. 
